\title{
The Libera generalized integral operator and Hardy spaces
}

\author{
Georghe Miclăus
}




\title{
THE LIBERA GENERALIZED INTEGRAL OPERATOR AND HARDY SPACES
}

\author{
GHEORGHE MICLĂUS
}

[Received: October 14, 2002]

\begin{abstract}
Aвstract. In this paper, we determine the Hardy spaces to which $L_{\gamma}^{n}(f)$ belongs, $n \in \mathbb{N}^{*}$, if $f \in H^{p}$, where $L_{\gamma}^{n}=\underbrace{L_{\gamma} \circ L_{\gamma} \circ \cdots \circ L_{\gamma}}$ is the Libera generalized integral operator (2). As a corollary we obtain the Hardy spaces for some classes of analytic functions.

Mathematics Subject Classification: 30C35, 30C45

Keywords: Hardy spaces, Libera generalized operator
\end{abstract}

\section{INTRODUCTION}

Let $f$ be an analytic function in the unit disc $U=\{z|| z \mid<1\}$ and let $H(U)$ denote the set of all analytic functions in $U$. In 1965, R. J. Libera [5] had studied the operator $L: H(U) \rightarrow H(U)$ defined by

$$
L(f)(z)=\frac{2}{z} \int_{0}^{z} f(t) d t
$$

showing that $L\left(S^{*}\right) \subset S^{*}$, where $S^{*}$ is the class of starlike functions, $L(K) \subset K$, where $K$ is the class of convex functions and $L(C) \subset C$, if $C$ is the class of close-to-convex functions. In 1969, S. D. Benardi generalized these results investigating in [1] a more general integral operator defined by $L_{\gamma}: H(U) \rightarrow H(U)$

$$
L_{\gamma}(f)(z)=\frac{\gamma+1}{z^{\gamma}} \int_{0}^{z} f(t) t^{\gamma-1} d t .
$$

He showed that if $\gamma \in \mathbb{N}^{*}$, then $L_{\gamma}$ preserves these properties. In this paper we determine the Hardy spaces, to which $L_{\gamma}^{n}(f)$ belongs, $n \in \mathbb{N}^{*}$, if $f \in H^{p}$, where $L_{\gamma}^{n}=\underbrace{L_{\gamma} \circ L_{\gamma} \circ \cdots \circ L_{\gamma}}_{n}$. 


\section{Preliminaries}

For $f \in H(U)$ and $z=r e^{i \theta}$, we set

$$
M_{p}(r, f)= \begin{cases}\left(\frac{1}{2 \pi} \int_{0}^{2 \pi}\left|f\left(r e^{i \theta}\right)\right|^{p} d \theta\right)^{\frac{1}{p}}, & 0<p<\infty \\ \sup _{0 \leq p \leq 2 \pi}\left|f\left(r e^{i \theta}\right)\right|, & \text { for } p=\infty .\end{cases}
$$

A function $f \in H(U)$ is said to be of Hardy spaces $H^{p}(0<p<\infty)$ if $M_{p}(r, f)$ remains bounded as $r \rightarrow 1^{-}$. $H^{\infty}$ is the class of bounded analytic functions in the unit disc.

We shall need the following well-known lemmas [2].

Lemma 1. If $f^{\prime} \in H^{p}, 0<p<1$, then $f \in H^{\frac{p}{1-p}}$. If $f^{\prime} \in H^{p}, 1 \leq p$, then $f \in H^{\infty}$.

Lemma 2. If $f \in H^{p}$ and $g \in H^{q}$, then $f g \in H^{\lambda}, \lambda=\frac{p q}{p+q}$.

Lemma 3 (Integral theorem of Hardy-Littlewood). If $f \in H^{p}$ and $F=\int_{0}^{z} f(t) d t$, then $f \in H^{\frac{p}{1-p}}$ for $0<p<1$, and $f \in H^{\infty}$ for $p \geq 1$.

\section{MaIn Results}

Let $\gamma \in C, \operatorname{Re} \gamma>0$, let $f$ be an analytic function in $U$ and let $L_{\gamma}$ be the Libera generalized integral operator (Bernardi's operator) defined by (2).

Theorem 1. If $f \in H^{p}$, then:

(i) if $n<\frac{1}{p}, n \in \mathbb{N}^{*}$, then $L_{\gamma}^{n}(f) \in H^{\lambda}, \lambda=\frac{p}{1-n p}$;

(ii) if $n \geq \frac{1}{p}, n \in \mathbb{N}^{*}$, then $L_{\gamma}^{n}(f) \in H^{\infty}$, where $L_{\gamma}^{n}=\underbrace{L_{\gamma} \circ L_{\gamma} \circ \cdots \circ L_{\gamma}}_{n}$;

(iii) $\left[L_{\gamma}(f)\right]^{\prime} \in H^{p}$.

Proof. Assertions ( $i)$ and (ii). If $f$ is analytic, $f(z)=z+a_{2} z^{2}+\ldots$, then there exists a unique function

$$
L_{\gamma}(f)(z)=\frac{\gamma+1}{z^{\gamma}} \int_{0}^{z} f(t) t^{\gamma-1} d t,
$$

which is analytic in $U$. Indeed,

$$
g(z)=\frac{f(z)}{z}=1+a_{2} z+\ldots
$$


is analytic in $U$. Let $h(z)=z[g(z)]^{\frac{1}{\gamma+1}}$ be the branch that is 1 for $z=0$, and we have that $h(z)=z+b_{2} z^{2}+\ldots$ is analytic in $U, \frac{h(z)}{z} \neq 0,[h(z)]^{\gamma+1}=\left(a_{2} z+\ldots\right)$. Hence

$$
\begin{aligned}
L_{\gamma}(f)(z) & =\frac{\gamma+1}{z^{\gamma}} \int_{0}^{z} f(t) t^{\gamma-1} d t=\frac{\gamma+1}{z^{\gamma}} \int_{0}^{z} \frac{h^{\gamma+1}(t)}{t} d t= \\
& =\frac{\gamma+1}{z^{\gamma}} \int_{0}^{z} t^{\gamma}\left(1+a_{2} t+\ldots\right) d t=z+\frac{\gamma+1}{\gamma+2} a_{2} z^{2}
\end{aligned}
$$

For the integral operator $L_{\gamma}$ we have $L_{\gamma}=A \circ B$, where

$$
A(f)(z)=\frac{\gamma+1}{z^{\gamma}} f(z), \quad \gamma \in C, \quad \operatorname{Re} \gamma>0
$$

and

$$
B(f)(z))=\int_{0}^{z} f(t) t^{\gamma-1} d t .
$$

We determine the Hardy class for $A$ and $B$. If $f \in H^{p}, \gamma=a+i b, a>0$, then

$$
M_{p}(r, A) \leq \frac{\gamma+1}{r^{a}}\left(\frac{1}{2 \pi} \int_{0}^{2 \pi}\left|f\left(r e^{i \theta}\right)\right|^{p} d \theta\right)^{\frac{1}{p}},
$$

and hence $A(f) \in H^{p}$.

From integral theorem of Hardy-Littlewood (Lemma 3), we have $B(f) \in H^{\frac{p}{1-p}}$ for $f \in H^{p}, p<1$, and $B(f) \in H^{\infty}$, for $f \in H^{p}, p \geq 1$. Because $L_{\gamma}(f)=A(B(f))$ we have for $f \in H^{p}, p<1$ that $B(f) \in H^{\frac{p}{1-p}}$ and $A(B(f)) \in H^{\frac{p}{1-p}}$. Hence $L_{\gamma}(f) \in H^{\frac{p}{1-p}}$ for $p<1$ and $L_{\gamma}(f) \in H^{\infty}$, for $p \geq 1$. Suppose that $n<\frac{1}{p}, n \in \mathbb{N}^{*}$ and $L_{\gamma}^{k}(f) \in H^{\frac{p}{1-k p}}$ for $1 \leq k \leq n-1$. Then

$$
L_{\gamma}^{n}(f) \in H^{\lambda}, \quad \lambda=\frac{\frac{p}{1-(n-1) p}}{1-\frac{p}{1-(n-1) p}}=\frac{p}{1-n p} .
$$

If $n \geq \frac{1}{p}$, then there is a $k \in \mathbb{N}^{*}$ such that $k<\frac{1}{p} \leq k+1 \leq n$ and $L_{\gamma}^{k}(f) \in H^{\frac{p}{1-k p}}$ and $L_{\gamma}^{k+1}(f) \in H^{\infty}$.

Assertion (iii). We have

$$
\left[L_{\gamma}(f)(z)\right]^{\prime}=\frac{1}{z}\left[(\gamma+1) f(z)-\gamma L_{\gamma}(f)(z)\right] .
$$


Applying the Minkowski inequality, we obtain

$$
\begin{aligned}
M_{p}^{p}\left(r,\left[L_{\gamma}(f)\right]\right) & =\frac{1}{2 \pi} \int_{0}^{2 \pi}\left|\left[L_{\gamma}(f)\left(r e^{i \theta}\right)\right]\right|^{p} d \theta= \\
& =\frac{1}{2 \pi} \int_{0}^{2 \pi} \frac{1}{\left|r e^{i \theta}\right|^{p}}\left|(\gamma+1) f\left(r e^{i \theta}\right)-\gamma L_{\gamma}(f)\left(r e^{i \theta}\right)\right|^{p} d \theta \leq \\
& \leq \frac{1}{2 \pi} \frac{|\gamma+1|^{p}}{r^{p}} \int_{0}^{2 \pi}\left|f\left(r e^{i \theta}\right)\right|^{p} d \theta+\frac{1}{2 \pi} \frac{|\gamma|^{p}}{r^{p}} \int_{0}^{2 \pi}\left|L_{\gamma}(f)\left(r e^{i \theta}\right)\right|^{p} d \theta .
\end{aligned}
$$

The value $M_{p}^{p}\left(r,\left[L_{\gamma}(f)\right]^{\prime}\right)$ is bounded for

$$
\min \left\{p, \frac{p}{1-p}\right\}=p
$$

Hence, $\left[L_{\gamma}(f)\right]^{\prime} \in H^{p}$. That results are best possible because from $\left[L_{\gamma}(f)\right]^{\prime} \in H^{q}$, $q>p$ applying (3) we should conclude that $f \in H^{q}$.

For $n=1$, these results were obtained in [4].

Remark 1. Since the results for Hardy classes do not depend on $\gamma$, the results remain the same for $L=L_{\gamma 1} \circ L_{\gamma 2} \circ \cdots \circ L_{\gamma n}$, where $\operatorname{Re}_{i}>0, i=\overline{1, n}$.

For $\gamma=1, L_{\gamma}=L$ is the Libera operator (1), and we have the following

Corollary 1. If $f$ is an analytic function in $U, \gamma \in \mathbb{C}, \operatorname{Re}_{i}>0$ and $f \in H^{p}$ then:

(i) if $n<\frac{1}{p}, n \in \mathbb{N}^{*}$, then $L^{n}(f) \in H^{\lambda}, \lambda=\frac{p}{1-n p}$;

(ii) if $n \geq \frac{1}{p}, n \in \mathbb{N}^{*}$, then $L^{n}(f) \in H^{\infty}$;

(iii) $\left[L^{n}(f)\right]^{\prime} \in H^{p}$.

Corollary 2. If $f \in K, f \neq \frac{z}{1-z e^{i \tau}}, \tau \in \mathbb{R}$ then $L_{\gamma}(f)$ is a bounded function.

It is well-known that if $f \in K$ (a convex function) then $f \in H^{1+\varepsilon}, \varepsilon=\varepsilon(f)>0$. Hence, we obtain from Theorem 1 that $L_{\gamma}(f) \in H^{\infty}$.

Therefore, $L_{\gamma}$ transforms $K$ into its subclass of bounded functions, excepting extremal functions.

Corollary 3. If $f \in S^{*}, f(z) \neq \frac{z}{\left(1+z e^{i \tau}\right)^{2}}$, then

(i) $L_{\gamma}(f) \in H^{1}$; 
(ii) $L_{\gamma}^{2}(f) \in H^{\infty}$ if $L_{\gamma}(f) \neq \frac{z}{\left(1+z e^{i \tau}\right)^{2}}, \tau \in \mathbb{R}$.

It is known that if $f \in S^{*}$, then $f \in H^{\frac{1}{2}+\varepsilon}, \varepsilon=\varepsilon(f)>0$. Hence $L_{\gamma}(f) \in H^{1}$. If

$$
L_{\gamma}(f)=\frac{z}{\left(1+z e^{i \tau}\right)}
$$

then $L_{\gamma}^{2}(f) \in H^{\infty}$.

In other words, $L_{\gamma}$ transforms $S^{*}$ into its subclass and $L_{\gamma}^{2}$ transforms $S^{*}$ into a subclass of bounded functions, with the exception of extremal functions.

Corollary 4. If $f \in \mathbb{C}, f(z) \neq \frac{p(z)}{\left(1+z e^{i \tau}\right)^{2}}$, Rep $(z)>0$, then

(i) $L_{\gamma}(f) \in H^{1}$;

(ii) $L_{\gamma}^{2}(f) \in H^{\infty}$ if $L_{\gamma}(f) \neq \frac{p(z)}{\left(1+z e^{i \tau}\right)}$, $\operatorname{Rep}(z)>0$.

It is known that if $f \in \mathbb{C}$ and $f(z) \neq \frac{p(z)}{\left(1+z e^{i \tau}\right)^{2}}, \operatorname{Re} p(z)>0$ then $f \in H^{\frac{1}{2}+\varepsilon}, \varepsilon=$ $\varepsilon(f)>0$. Hence, $L_{\gamma}(f) \in H^{1}$. If $L_{\gamma}(f) \neq \frac{p(z)}{\left(1+z e^{i \tau}\right)}, \operatorname{Re} p(z)>0$ from Theorem 1 we obtain $L_{\gamma}^{2}(f) \in H^{\infty}$.

\section{REFERENCES}

[1] Bernandi, S. D.: Convex and starlike univalent functions, Trans. Amer. Math. Soc., 135, (1969), 429-446.

[2] Duren, P. L.: Theory of $H^{p}$ spaces, Academic Press, New York and London, 1970.

[3] Eenigenburg, P. J. and Keogh, F.R.: The Hardy classes of some univalent functions and their derivatives, Mich. Math. J., 17, (1970), 335-346.

[4] Fenete, O.: Some integral operators and Hardy spaces, Mathematica (Cluj), 29(52), (1987), 29-31.

[5] Libera R.J.: Some classes of regular univalent functions, Proc. Amer. Math. Soc., 16, (1965), 75558 .

[6] Miller, S. S. andMocanu, P. T. and Reade, M. O.: The Hardy classes for functions in the class $M V[a, k]$, J. Math. Anal. Appl., 51, (1975), 33-42.

\section{Author's Address}

\section{Gheorghe Miclăus:}

Department of Mathematics and Computer Science, North University of Baia Mare, Romania

E-mail address: miclaus5@yahoo.com 